\title{
Routine gastric volume monitoring thrown out
}

Published online: 22 January 2013

(C) Springer Healthcare 2013

medwireNews: Monitoring gastric residual volumes (GRV) in critically ill patients receiving enteral nutrition does not prevent them from developing ventilator-associated pneumonia (VAP), shows a randomized trial.

Patients who were not monitored vomited more often than those who were, but this did not affect any other outcomes. Also, they were more likely to achieve their goal calorie intake.

The findings "should instill confidence in clinicians to change practice and not routinely check GRVs in all patients mechanically ventilated receiving enteral nutrition," says Todd Rice (Vanderbilt University School of Medicine, Nashville, Tennessee, USA) in an editorial accompanying the study in JAMA.

He notes: "Despite emerging evidence to the contrary, many enteral feeding protocols continue to interrupt enteral feeding for relatively low GRVs, some with thresholds as low as $150 \mathrm{~mL}$ or twice the enteral feeding rate the patient is receiving at the time."

The researchers, led by Jean Reignier (District Hospital Center, La Roche-sur-Yon, France), adopted $250 \mathrm{~mL}$ as the GRV that prompted them to withhold feeding in the 222 patients in the control group, whose GRVs were monitored every 6 hours.

VAP occurred in $15.8 \%$ of these patients, and also in $16.7 \%$ of the 227 patients who were not monitored, with feeding interrupted only if they vomited. The difference was not significant, which Rice says "supports the emerging concept that aspiration of oropharyngeal secretions plays a larger role in the development of VAP than gastric or gastrointestinal contents."

Patients whose GRVs were not monitored more often vomited, at 39.6\% compared with $27.0 \%$ of the control group, but because vomiting also defined intolerance to enteral feeding, this was diagnosed much less often than in the control group, for whom it was defined as a GRV of $250 \mathrm{~mL}$ or more and occurred in $63.5 \%$.

Consequently, enteral feeding was interrupted less frequently in the unmonitored patients and they were significantly more likely to achieve their calorie goals. Also, the team used an aggressive feeding protocol, rather than a "ramp-up" schedule, so around 70\% of all patients were at their calorie goal after the first day. 
But despite receiving more calories, unmonitored patients fared no better nor worse than the monitored group in terms of outcomes including the duration of mechanical ventilation, length of stay in the intensive care unit and in hospital, and mortality within 28 and 90 days.

"Thus, the time and energy that health care practitioners expend on trying to rapidly achieve goal enteral feeding rates early in the course of critical illness may be better spent on other aspects of critical care," suggests Rice.

By Eleanor McDermid, Senior medwireNews Reporter

\section{Reference}

JAMA 2013; 309: 249-256, 283-284 